\title{
The ATLAS Hardware Track Trigger performance studies for the HL-LHC
}

\section{Ana Luísa Carvalho* on behalf of the ATLAS Collaboration}

LIP - Laboratório de Instrumentação e Física Experimental de Partículas, Lisbon, Portugal

E-mail: analuisamc@tecnico.ulisboa.pt

For the High-Luminosity LHC, planned to start in 2027, the ATLAS experiment will be equipped with the Hardware Tracking for the Trigger (HTT) system, a dedicated hardware system able to reconstruct tracks in the silicon detectors with short latency. The evolved trigger and data acquisition system design consists of a two-level hardware trigger in which the HTT is used in a low-latency mode (L1Track), providing tracks in regions of ATLAS at a rate of up to $4 \mathrm{MHz}$, with a latency of a few tens of micro-seconds. Different readout scenarios for the silicon pixel layers of the inner detector are under study. While the full simulation of HTT is under development, an emulation procedure is developed to study the impact of L1Track-like tracking resolutions on physics benchmark channels. In this document we describe the status of the ongoing HTT performance studies for the HL-LHC trigger menu, covering the impact on $b$-tagging and on multi-jet and leptonic trigger selections.

The Eighth Annual Conference on Large Hadron Collider Physics-LHCP2020

25-30 May, 2020

online

\footnotetext{
${ }^{*}$ Speaker
} 


\section{Introduction and motivation}

The High-Luminosity LHC (HL-LHC) project [1], planned to start in 2027, will increase the LHC instantaneous luminosity by a factor five to seven with respect to the design value. In the ATLAS experiment [2], at full luminosity, an average of 140 to 200 proton-proton collisions per bunch crossing (pileup) are expected, compared to around 60 in Run 2. Given the current uncertainty in the projected trigger rates for selections including jets and the uncertainty in the occupancy in the inner silicon detector layers, the trigger and data acquisition system needs to be flexible enough to accommodate increased rates and occupancies. A two-level hardware trigger mitigates both of these risks and it is under study as an alternative to the baseline single-level system. It includes a Level-0 (L0) system that reduces event throughput from $40 \mathrm{MHz}$ to a maximum of $4 \mathrm{MHz}$, and a Level-1 (L1) system that further reduces the rate to less than $1 \mathrm{MHz}$. In this architecture, the L1 system uses hits in the inner tracker strip detector and the outer layers of the pixel detector (ITk) to build tracks within regions identified by the L0 trigger with $p_{\mathrm{T}}>4 \mathrm{GeV}$ (L1Track). This provides rudimentary primary vertex and impact parameter selections that can be particularly useful to handle the increased acceptance of hadronic trigger selections at Level-0.

While the full simulation of the L1Track system is under development, an emulation procedure is developed in order to study the impact of L1Track-like tracking resolutions on physics benchmark channels. The details of the L1Track emulation are presented in section 2. The results are shown in sections 3, 4 and 5 for a multi-jet, di-muon and $b$-tagged jet selections, respectively.

\section{L1Track emulation}

The expected performance of the L1Track system is emulated starting from offline-reconstructed tracks from which few ITk hits are dropped in order to reproduce the L1Track system with eight layers. Tracks are then refitted with only those ITk hits using the same high-precision offline trackfitting algorithm [3]. According to which layers are used to perform the refit, three scenarios are considered: (1) Offline: all ITk layers are used, (2) TDR ${ }^{1}$ : only the outer pixel and strip layers are used and (3) Strip-only: only the strip layers are used. Offline tracking is used only as a reference since it is not expected that track reconstruction at Level-1 would ever achieve the performance of offline full-detector precision tracking.

Based on the track refitting procedure, the resolutions of the track perigee parameters $z_{0}{ }^{2}$ and $d_{0}{ }^{3}$ are derived. An additional factor two is introduced to account for differences between online and offline track reconstruction and a $95 \%$ track reconstruction efficiency is emulated by randomly dropping $5 \%$ of the tracks. The resolution of the $z_{0}$ parameter is shown in Figure 1a.

The studies shown here do not take into account the contribution from fake tracks and use quantities reconstructed with offline algorithms. Therefore, they should be taken as an upper limit to what tracking at the L1 could achieve in terms of rejection with respect to the L0 system. The Monte Carlo samples used are simulated with $\sqrt{s}=14 \mathrm{TeV}$ and $\langle\mu\rangle=200$, using ATLAS full simulation with ITk layout Step 2.2 [3]. Tracks are obtained using the L1Track emulation. Unless

\footnotetext{
${ }^{1}$ This scenario tries to reproduce the configuration used in the studies presented in the Phase-II TDAQ TDR [4].

${ }^{2} z$ coordinate of the point of closest approach to the $z$-axis.

${ }^{3}$ Signed distance of closest approach to the $z$-axis.
} 
otherwise stated, multi-jet events with low $p_{\mathrm{T}}$ threshold are used as background in all studies and the rejection factors are calculated with respect to the corresponding $\mathrm{L} 0$ selection.

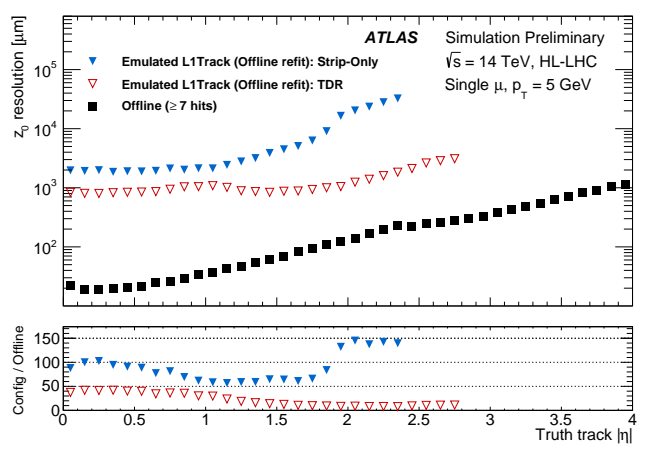

(a) Emulated L1Track resolution of the $z_{0}$ track parameter as a function of the truth track $\eta$ for single muons with $p_{\mathrm{T}}=5 \mathrm{GeV}$.

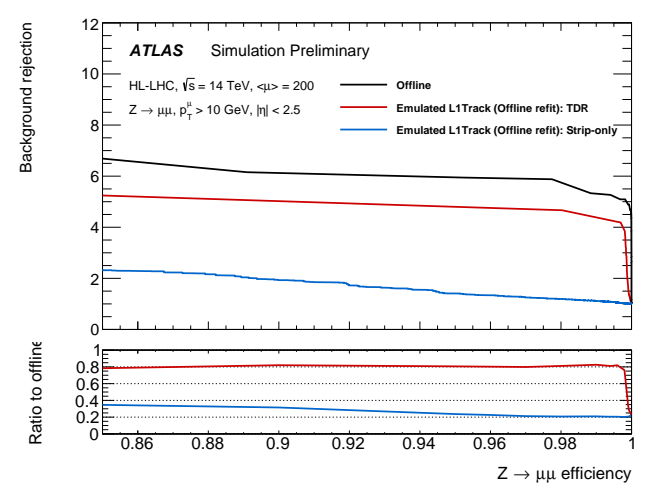

(c) Background rejection versus $Z \rightarrow \mu \mu$ signal efficiency for events with $\geq 2$ muons. The discriminant variable is the difference between the $z_{0}$ of the tracks matched to the muons.

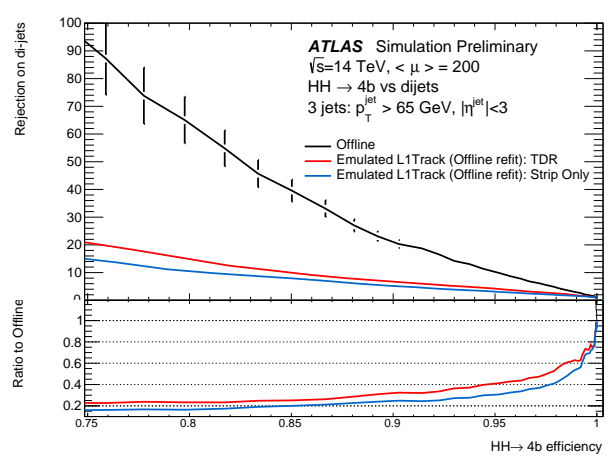

(b) Background rejection versus $H H \rightarrow 4 b$ signal efficiency for events with $\geq 3$ jets with $p_{\mathrm{T}}>3$ $\mathrm{GeV}$ and $|\eta|<3$. The discriminant variable is the output of a BDT that combines variables based on $z_{0}, d_{0}$ and track multiplicity inside jets.

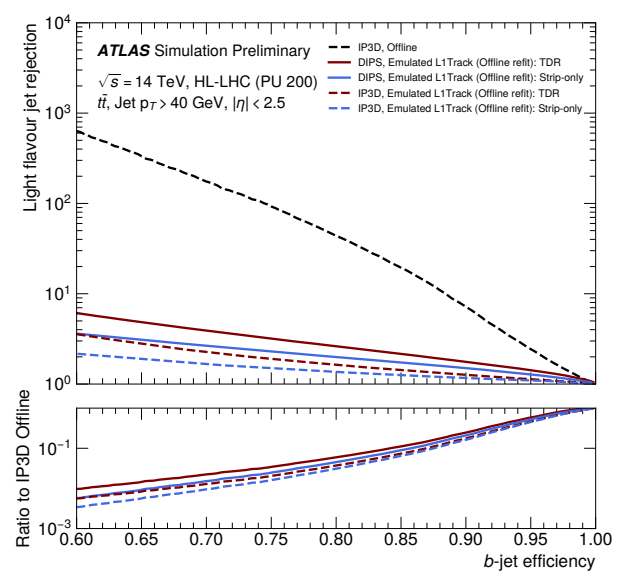

(d) Light-flavour jet rejection versus b-jet efficiency for the IP3D (dashed lines) and DIPS (solid lines) $b$-tagging algorithms trained on jets from $t \bar{t}$ events with $p_{\mathrm{T}}>40 \mathrm{GeV}$ and $|\eta|<2.5$.

Figure 1: Expected performance of the L1Track system based on an offline emulation for the TDR (red) and Strips-only (blue) scenarios [5]. The "Offline" scenario (black) uses unmodified offline tracks.

\section{Multi-jet triggers studies}

Multi-jet triggers with a low energy threshold are used, among others, in searches for $H H \rightarrow 4 b$ which is a key benchmark channel for HL-LHC. Common vertex requirements can help reduce the rate from background processes, thereby increasing the sensitivity to the signal process. The trigger 
selection requires at least three jets with $p_{\mathrm{T}}>65 \mathrm{GeV}$ and $|\eta|<3^{4}$ and a common vertex. Tracks are associated to jets in a cone. For each jet, the $z_{0}$ and $d_{0}$ parameters are computed from the $p_{\mathrm{T}}$ weighted sum of the tracks associated with the jet. A boosted decision tree (BDT) is trained using ten variables based on $z_{0} / d_{0}$ separation and track multiplicity. The output distribution of this BDT is used as the discriminant variable to plot the background rejection versus $H H \rightarrow 4 b$ signal efficiency, as shown in Figure 1b. At 85\% signal efficiency, a factor 10 (9) rejection can be achieved for the TDR (Strip-only) scenarios.

\section{Di-muon triggers studies}

Lowering the $p_{\mathrm{T}}$ thresholds in multi-lepton selections is key to keeping/increasing the acceptance in channels such as $H \rightarrow \tau \tau$ and SUSY searches. Here, the $Z \rightarrow \mu \mu$ process is used as signal. The trigger selection requires at least two muons with $p_{\mathrm{T}}>10 \mathrm{GeV}$ and a common vertex. The two leading muons are associated to the highest $p_{\mathrm{T}}$ track in a cone of $\Delta R<0.1^{5}$. The L0 trigger efficiency is emulated by applying a weight based on the efficiency of a muon trigger with a $p_{\mathrm{T}}$ threshold of $10 \mathrm{GeV}$. The distance along the $z$-axis between the two muons is used as discriminant variable to plot the background rejection versus $Z \rightarrow \mu \mu$ signal efficiency, as shown in Figure 1c. At $96 \%$ signal efficiency a factor 4.5 (1.5) rejection can be achieved for TDR (Strip-only) scenarios.

\section{B-tagged jets studies}

Explicit $b$-jet identification in multi-jet triggers at the Level-1 can help reduce the rate from hadronic processes with light-flavor jets. The trigger selection requires at least one jet with $p_{\mathrm{T}}>40$ $\mathrm{GeV}$ and $|\eta|<2.5$ and passing $b$-jet selection criteria with a specific tagging algorithm. The signal is identified with $b$-jets from $t \bar{t}$ events while the background are light-flavor jets also from $t \bar{t}$ events. Simplified versions of offline $b$-jet tagging methods are used to demonstrate the rejection power of such algorithms with a reduced number of layers available for track fitting. Two algorithms are studied: IP3D [6] and DIPS [7]. DIPS outperforms IP3D by using extra track-based kinematic inputs and by exploiting correlations between tracks. Both taggers have been modified to not use track hit content knowledge, as this information is not simulated in the current L1Track emulation.

Figure 1d shows the light-flavor jet rejection versus $b$-jet efficiency for IP3D (dashed lines) and DIPS (solid lines). For a 70\% $b$-jet efficiency a factor 4 (2.5) rejection can be achieved for the TDR (Strip-only) scenarios with the DIPS algorithm.

\section{Conclusion}

The studies presented in this document support the conclusion that using the outermost pixel layers in addition to the strip layers (TDR scenario) can improve background rejection with respect to the Level-0 rate by a factor 10 in multi-jet selections, 4.5 in di-muon selections and 4 in $b$-jet trigger selections. Nonetheless, the Strip-only scenario can still add sizeable background rejection to help facing the HL-LHC era.

\footnotetext{
${ }^{4}$ The pseudorapidity, $\eta$, is defined in terms of the polar angle $\theta$ as $\eta=-\ln (\theta / 2)$. The azimuthal angle around the $z$ axis (that lies along the beam pipe) is denoted by $\phi$.

${ }^{5} \Delta R=\sqrt{\Delta \phi^{2}+\Delta \eta^{2}}$
} 


\section{References}

[1] Apollinari G., Béjar Alonso I., Brüning O., Fessia P., Lamont M., Rossi L., and Tavian L. HighLuminosity Large Hadron Collider (HL-LHC): Technical Design Report V. 0.1. CERN Yellow Reports: Monographs. CERN, Geneva, 2017. https: //cds . cern. ch/record/2284929.

[2] ATLAS Collaboration. The ATLAS Experiment at the CERN Large Hadron Collider. JINST 3 (2008) S08003. https://cds. cern.ch/record/1129811.

[3] ATLAS Collaboration. Technical Design Report for the ATLAS Inner Tracker Pixel Detector. Technical Report CERN-LHCC-2017-021. ATLAS-TDR-030, CERN, Geneva, Sep 2017. https://cds. cern.ch/record/2285585.

[4] ATLAS Collaboration. Technical Design Report for the Phase-II Upgrade of the ATLAS TDAQ System. Technical Report CERN-LHCC-2017-020. ATLAS-TDR-029, CERN, Geneva, Sep 2017. https://cds. cern. ch/record/2285584.

[5] Approved plots for the L1Track Trigger project. https://twiki.cern.ch/twiki/bin/ view/AtlasPublic/L1TrackPublicResults\#0ffline_refit_L1Track_algorithm.

Accessed: 2020-08-03.

[6] ATLAS Collaboration. ATLAS b-jet identification performance and efficiency measurement with $t \bar{t}$ events in pp collisions at $\sqrt{s}=13 \mathrm{TeV}$. Eur. Phys. J. C 79 (2019) 970. hhttps: //cds. cern. ch/record/2682119/files/scoap3-fulltext . pdf?version=2.

[7] ATLAS Collaboration. Deep Sets based Neural Networks for Impact Parameter Flavour Tagging in ATLAS. Technical Report ATL-PHYS-PUB-2020-014, CERN, Geneva, May 2020. https: //cds. cern. ch/record/2718948. 